PRL

Submitted

\title{
Retardation of the Onset of Turbulence by Minor Viscosity Contrasts
}

\author{
Rama Govindarajan ${ }^{\dagger, *}$, Victor S. L'vov* and Itamar Procaccia* \\ * Dept. of Chemical Physics, The Weizmann Institute of Science, Rehovot 76100, Israel \\ $\dagger$ Fluid Dynamics Unit, Jawaharlal Nehru Centre for Advanced Scientific Research, Jakkur, Bangalore 560064, India.
}

\begin{abstract}
Motivated by the large effect of turbulent drag reduction by minute concentrations of polymers we study the effects of minor viscosity contrasts on the stability of hydrodynamic flows. The key player is a localized region where the energy of fluctuations is produced by interactions with the mean flow (the "critical layer"). We show that a layer of weakly space-dependent viscosity placed near the critical layer can have very large stabilizing effect on hydrodynamic fluctuations, retarding significantly the onset of turbulence. The effect is not due to a modified dissipation (as is assumed in theories of drag reduction), but due to reduced energy intake from the mean flow to the fluctuations. We propose that similar physics act in turbulent drag reduction.
\end{abstract}

The addition of small amounts of polymers to hydrodynamic systems produces dramatic effects on phenomena such as the transition to turbulence, vortex formation and turbulent transport [1]. The phenomenon that attracted most attention was, for obvious reasons, the reduction of friction drag by up to $80 \%$ when a very small concentration of long-chain polymers were added to turbulent flows [2] 3. In spite of the fact that the phenomenon is robust and the effect huge, there exists no accepted theory that can claim quantitative agreement with the experimental facts. Moreover, it appears that there is no mechanistic explanation. In the current theory that is due to de Gennes [4.5] one expects the Kolmogorov cascade to be terminated at scales larger than Kolmogorov scale, leading somehow to an increased buffer layer thickness and reduced drag, but how this happens and what is the fate of the turbulent energy is not being made clear.

In this Letter we propose that the crucial issue is in the production of energy of hydrodynamic fluctuations by their interaction with the mean flow. For the sake of concreteness we examine a simple laminar flow and its loss of stability, and show how small viscosity contrasts lead to an order of magnitude retardation in the onset of instability of "dangerous" disturbances. In this model everything is explicitly calculable, and we demonstrate that nothing special happens to the dissipation. Rather, it is the energy production that is dramatically reduced, giving rise to a large effect for a small cause. At the end of this Letter we argue that similar physics may very well be at the heart of turbulent drag reduction, but we stress that the phenomenon discussed below is interesting by itself and well warrants an experimental confirmation.

It is well known that parallel Poiseuille flow loses its stability at some threshold Reynolds number $R e=R e_{t h}$ (close to 5772). It is also well known that the instability is "convective", with the most unstable mode having a phase velocity $c$. Analytically it has the form

$$
\hat{\phi}(x, y, t)=\frac{1}{2}\{\phi(y) \exp [\mathrm{i} k(x-c t)]+\text { c.c. }\} \exp (\gamma t),
$$

where $\hat{\phi}(x, y, t)$ is the disturbance streamfunction and $\phi(y)$ is the complex envelope of $\hat{\phi}(x, y, t)$. We have chosen

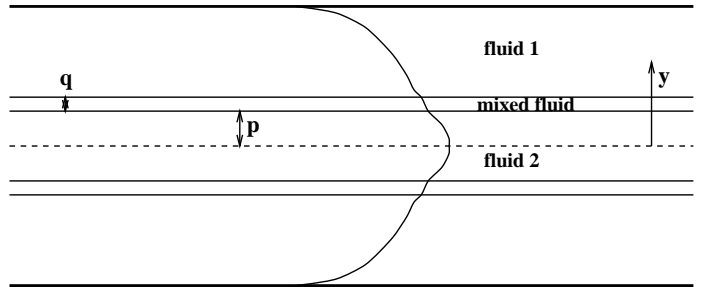

FIG. 1. Schematic of the flow: the fluid near the walls has a viscosity $\mu_{1}$, and that flowing at the center is of viscosity $\mu_{2}$. In the mixed layer (of width $q$ ) the viscosity varies gradually between $\mu_{1}$ and $\mu_{2}$. The parameter $p$ controls the position of the mixed layer.

$x$ and $k$ as the streamwise coordinate and wavenumber of the disturbance, $c$ as the phase speed and $t$ as time. $\gamma$ is the increment of instability. What is not usually emphasized is that the main interactions leading to the loss of stability occur in a sharply defined region in the channel, i.e. at a layer whose distance from the wall is such that the phase velocity $c$ is comparable to the velocity of the mean flow. We refer to this layer as the "critical" layer. It is thus worthwhile to examine the effect on the stability of Poiseuille flow of a viscosity gradient placed in the vicinity of the critical layer. Following [6] we examine a channel flow of two fluids with different viscosities $\mu_{1}$ and $\mu_{2}$, see Fig. 11.

Observing that the inferred effective viscosity in polymer drag reduction increases towards the center by about $30 \%$ over about a $1 / 3$ of the half-channel [7], we choose $\mu_{2}=1$ and $m \equiv \mu_{1} / \mu_{2}=0.9$, with all the viscosity difference of 0.1 concentrated in a "mixing" layer of width 0.1 , leading to comparable viscosity gradients. The observation that we want to focus on is shown in Fig. 2: the threshold Reynolds number for the loss of stability of the mode as in Eq. (1) depends crucially on the position of the mixing layer. When the latter hits the critical layer the threshold Reynolds number for the loss of stability reaches as much as 88000 . In other words, one can increase the threshold of instability for a given mode 15 times, and by making the mixing layer thinner one can reach even higher threshold Reynolds values. 


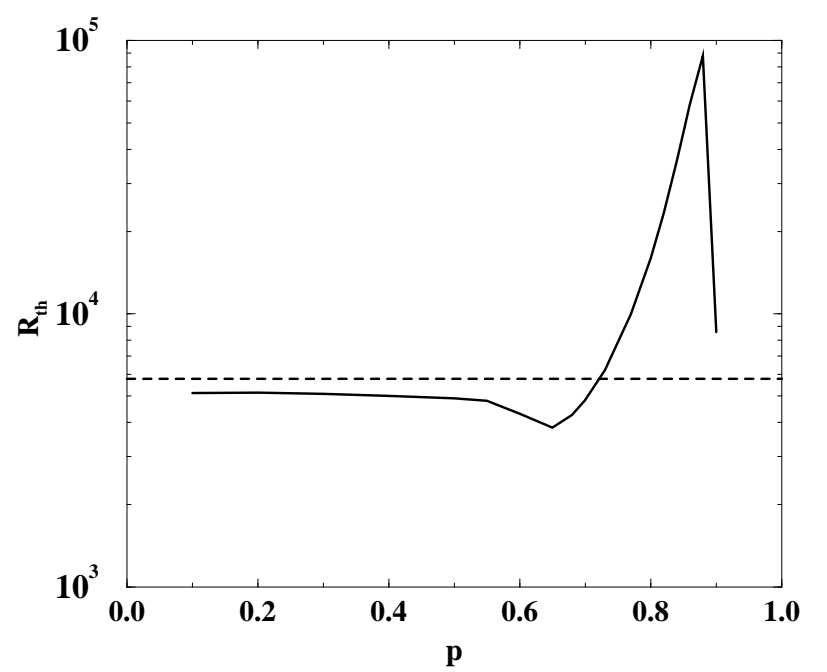

FIG. 2. The dependence of the threshold Reynolds number on the position of the viscosity stratified layer for $m=0.9$. The dashed line pertains to the neat fluid. Note the huge increase in $R_{\mathrm{th}}$ within a small range. This occurs when the stratified layer overlaps the critical layer.

In this Letter we analyze the physical origin of this huge sensitivity of the flow stability to the profile of the viscosity. The stability of this flow is governed by the modified Orr-Sommerfeld equation [8]

$$
\begin{aligned}
& \mathrm{i} k\left[\left(\phi^{\prime \prime}-k^{2} \phi\right)(U-c-\mathrm{i} \gamma)-U^{\prime \prime} \phi\right]=\frac{1}{\operatorname{Re}}\left[\mu \phi^{(4)}+2 \mu^{\prime} \phi^{\prime \prime \prime}\right. \\
& \left.+\left(\mu^{\prime \prime}-2 k^{2} \mu\right) \phi^{\prime \prime}-2 k^{2} \mu^{\prime} \phi^{\prime}+\left(k^{2} \mu^{\prime \prime}+k^{4} \mu\right) \phi\right]
\end{aligned}
$$

in which $\phi, U$ and $\mu$ are functions of $y$. The boundary conditions are $\phi( \pm 1)=\phi^{\prime}( \pm 1)=0$. All quantities have been non-dimensionalised using the half-width $H$ of the channel and the centerline velocity $U_{0}$ as the length and velocity scales respectively. The Reynolds number is defined as $\operatorname{Re} \equiv \rho U_{0} H / \mu_{2}$, where $\rho$ is the density (equal for the two fluids). The primes stand for derivative with respect to $y$. At $y=0$, we use the even symmetry conditions $\phi(0)=1$ and $\phi^{\prime}(0)=0$, as the even mode is always more unstable than the odd.

Since the flow is symmetric with respect to the channel centerline, we restrict our attention to the upper halfchannel. Fluid 2 occupies the region $0 \leq y \leq p$. Fluid 1 lies between $p+q \leq y \leq 1$. The region $p \leq y \leq p+q$ contains mixed fluid. The viscosity is described by a steady function of $y$, scaled by the inner fluid viscosity $\mu_{2}$ :

$\mu(y)=1, \quad$ for $\quad 0 \leq y \leq p$,

$\mu(y)=1+(m-1) \xi^{3}\left[10-15 \xi+6 \xi^{2}\right], 0 \leq \xi \leq 1$,

$\mu(y)=m, \quad$ for $\quad p+q \leq y \leq 1$,

Here $\xi \equiv(y-p) / q$ is the mixed layer coordinate. We have assumed a 5 th-order polynomial profile for the viscosity in the mixed layer, whose coefficients maintain the

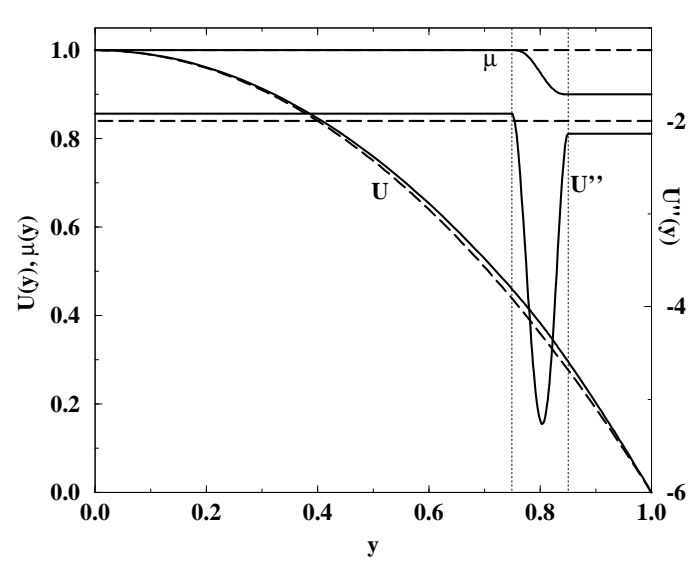

FIG. 3. Profiles of the normalized viscosity $\mu(y)$ and normalized velocity $U(y)$ and the second derivative $U^{\prime \prime}(y)$ for $m=0.9$ (solid lines) and $m=1.0$ (dashed lines). The mixed layer is between the vertical dashed lines.

viscosity and its first two derivatives continuous across the mixed layer. The exact form of the profile is unimportant. For a plot of the profile $m=0.9$, see Fig. 3 .

The basic flow $U(y)$ is obtained by requiring the velocity and all relevant derivatives to be continuous at the edges of the mixed layer:

$$
\begin{array}{cll}
U(y)=1-G y^{2} / 2, & \text { for } \quad y \leq p, \\
U(y)=U(p)-G \int_{p}^{y} \frac{y}{\mu} \mathrm{d} y, & \text { for } \quad p \leq y \leq p+q, \\
U(y)=G\left(1-y^{2}\right) / 2 m, & \text { for } \quad y \geq p+q .
\end{array}
$$

Here $G$ is the streamwise pressure gradient.

It can be seen, comparing the mean profile $U(y)$ to that of the neat fluid (cf. Fig. 3), that nothing dramatic happens to this profile even when the mixing layer is chosen to overlap a typical critical layer. Accordingly we need to look for the origin of the large effect of Fig. 2 in the energetics of the disturbances. To do so, recall that the streamwise and normal components of the disturbance velocity $\hat{u}(x, y, t)$ and $\hat{v}(x, y, t)$ may be expressed via streamfunction as usual:

$$
\hat{u}(x, y, t)=\partial \hat{\phi} / \partial y \quad \text { and } \quad \hat{v}(x, y, t)=-\partial \hat{\phi} / \partial x
$$

These functions may be written in terms of complex envelopes similar to Eq. (11):

$$
\begin{aligned}
& \hat{u}(x, y, t)=\frac{1}{2}\{u(y) \exp [\mathrm{i} k(x-c t)]+\text { c.c. }\} \exp (\gamma t), \\
& \hat{v}(x, y, t)=\frac{1}{2}\{v(y) \exp [\mathrm{i} k(x-c t)]+\text { c.c. }\} \exp (\gamma t) .
\end{aligned}
$$

The pressure disturbance $\hat{p}$ is defined similarly.

Define now a disturbance of the density of the kinetic energy

$$
\hat{E}(x, y, t)=\frac{1}{2}\left[\hat{u}(x, y, t)^{2}+\hat{v}(x, y, t)^{2}\right]
$$


we can express the mean (over $x$ ) density of the kinetic energy as follows:

$$
\begin{aligned}
E(y, t) & \equiv\langle\hat{E}(x, y, t)\rangle_{x}=\mathcal{E}(y) \exp (2 \gamma t), \\
\mathcal{E}(y) & =\frac{1}{4}\left(|u(y)|^{2}+|v(y)|^{2}\right) .
\end{aligned}
$$

The physics of our phenomenon will be discussed in terms of the balance equation for the averaged disturbance kinetic energy. Starting from the linearized NavierStokes equations for $\hat{u}$ and $\hat{v}$, dotting it with the disturbance velocity vector, averaging over one cycle in $x$ and using Eqs. (11)-(13) leads to

$$
2 \gamma \mathcal{E}(y)=\nabla \cdot J(y)+W_{+}(y)-W_{-}(y),
$$

where the energy flux $J(y)$ in the $y$ direction, rates of energy production (by the mean flow) $W_{+}(y)$ and energy dissipation (by the viscosity) $W_{-}(y)$ are given by

$$
\begin{aligned}
J(y) & \equiv \frac{\left[u(y) p^{*}(y)+\text { c.c. }\right]}{4 \rho}+\frac{1}{\operatorname{Re}} \mu(y) \nabla \mathcal{E}(y), \\
W_{+}(y) & \equiv-\frac{1}{4} U^{\prime}(y)\left[u(y) v^{*}(y)+\text { c.c. }\right], \\
W_{-}(y) & \equiv \frac{\mu(y)}{\operatorname{Re}}\left\{2 k^{2} \mathcal{E}(y)+\frac{1}{2}\left[\left|u^{\prime}(y)\right|^{2}+\left|v^{\prime}(y)\right|^{2}\right]\right\} .
\end{aligned}
$$

The superscript $*$ denotes the complex conjugate. To plot these functions we need to solve Eq. (2) as an eigenvalue problem, to obtain $c, \gamma$, and $\phi(y)$ at given Re and $k$. The value of $c$ determines the position of the critical layer.

It is convenient to compute and compare the space averaged production and dissipation terms $\Gamma_{+}$and $\Gamma_{-}$ defined by:

$$
\Gamma_{ \pm} \equiv \int_{0}^{1} W_{ \pm}(y) \mathrm{d} y / \int_{0}^{1} \mathcal{E}(y) \mathrm{d} y .
$$

The local production of energy can be positive or negative, indicative of energy transfer from the mean flow to the disturbance and vice-versa respectively. The production in one region (where $W_{+}(y)>0$ ) can be partly canceled out by a "counter-production" in other region (where $W_{+}(y)<0$ ).

The use of these measures can be exemplified with the neat fluid ( $m=1.0$ here). The laminar flow displays its first linear instability at a threshold Reynolds number of $R e_{\text {th }}=5772$, which means that the total production $\Gamma_{+}$across the layer becomes equal to the total dissipation $\Gamma_{-}$at this value of Re. Examining Fig. 1 we can see that the disturbance kinetic energy is produced predominantly within the critical layer, where the basic flow velocity is close to the phase speed of the disturbance, while most of the dissipation is in the wall layer.

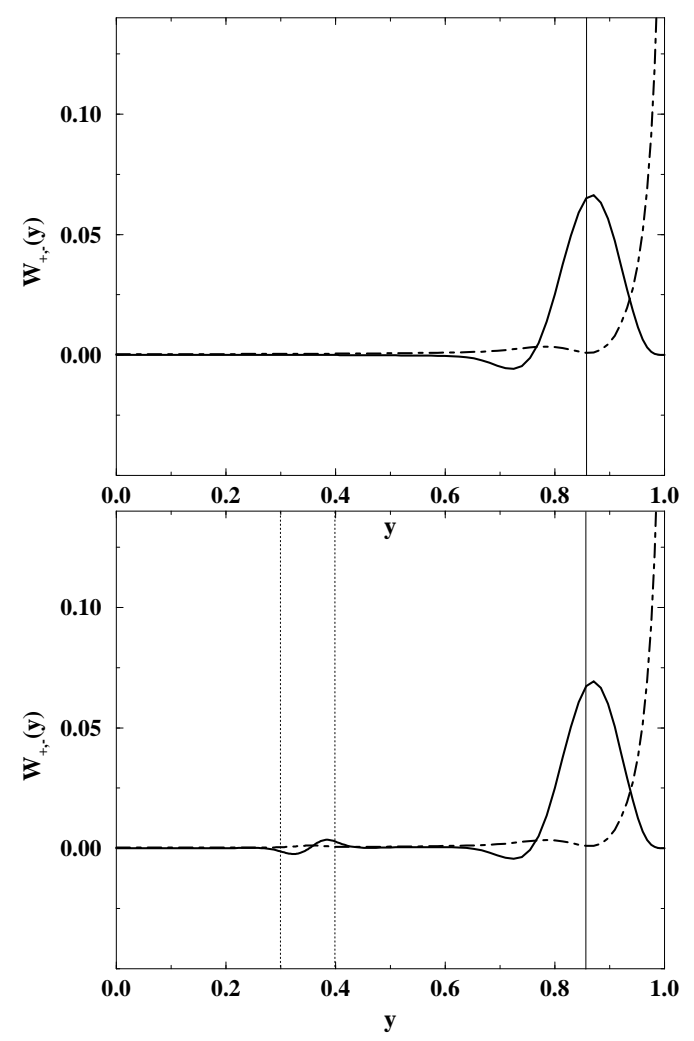

FIG. 4. Energy balance: production $W_{+}(y)$, solid line; dissipation $W_{-}(y)$, dot-dashed line, $\operatorname{Re}=5772$. Top: $m=1$, $\Gamma_{+}=\Gamma_{-}=0.0148$. Bottom: $m=0.9, p=0.3, \Gamma_{+}=0.0158$, $\Gamma_{-}=0.0148$. In this and all the subsequent figures the solid vertical lines show the location of the critical lines, whereas the region between the dotted lines is the mixed layer.

The balance is not changed significantly when the viscosity ratio is changed to 0.9 so long as the mixed layer is not close to the critical layer. There is a small region of production and one of counter-production within the mixed layer, whose effects cancel out, leaving the system close to marginal stability.

We now turn our attention to Fig. 5, in which the main point of this Letter is demonstrated. Here, the Reynolds number is the same as before, but the mixing layer has been moved close to the critical layer. It is immediately obvious that the earlier balance is destroyed. The counter-production peak in the mixed layer is much larger than before, making the flow more stable. The wavenumber used is that at which the flow is least stable for the given Reynolds number at this $p$. For $m=0.9$, the threshold Reynolds number is 46400 . Fig. ?? shows the energy balances at marginal stability - the picture is qualitatively the same here as at $R e \approx 5772$ for the neat fluid. 

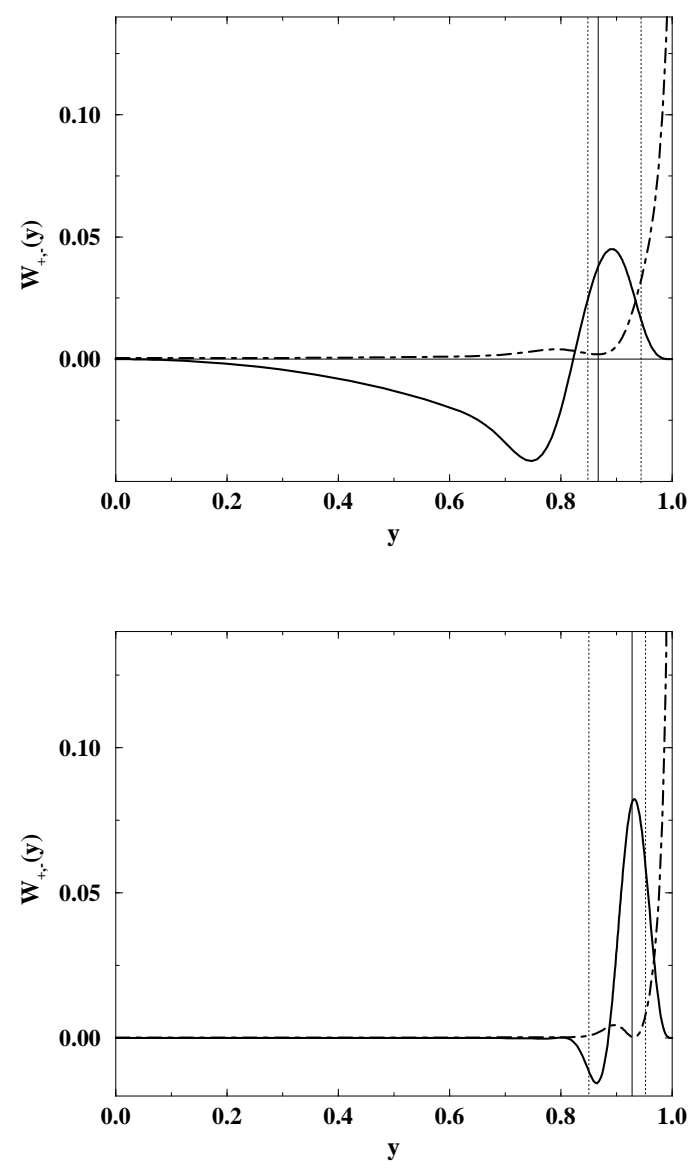

FIG. 5. Energy balance for $m=0.9, p=0.85$. Energy roduction $W_{+}(y)$, solid line; dissipation $W_{-}(y)$, dot-dashed line. Upper panel: stable flow at $\operatorname{Re}=5772$ (with $\Gamma_{+}=-0.0114$, $\left.\Gamma_{-}=0.0122\right)$. Lower panel: Marginal flow at $\operatorname{Re}=46400$ (with $\Gamma_{+}=\Gamma_{-}=0.0053$ ).

The main factor determining the stability is the production, which is driven by the phase change caused by the viscosity stratification. The dissipation on the other hand depends only on Reynolds number and does not respond disproportionately to changes in viscosity. In neat fluids, the term containing $U^{\prime \prime}(y)$ in (2) is always of higher order within the critical layer. However, with the introduction of a viscosity gradient within the critical layer, the gradients of the basic velocity profile will scale according to the mixed layer coordinate $\xi$. An analysis in the critical layer indicates that for $q \leq O\left(R e^{-1 / 3}\right)$, the term containing $U^{\prime \prime} /(U-c)$ is now among the most dominant. Any reasonable viscosity gradient of the right sign will pick up this term, leading to vastly enhanced stability. Note the dramatic effect in $U^{\prime \prime}$ in Fig. 3 .

Indeed, in the light of this discussion we can expect that the large effect of retardation of the instability would even increase if we make the mixing layer thinner. This is indeed so. Nevertheless, one cannot conclude that instability can be retarded at will, since other disturbances, differing from the primary mode, become unstable first, albeit at a much higher Reynolds number than the primary mode; when we stabilize a given mode substantially, we should watch out for other pre-existing/newly destabilized modes which may now be the least stable.

Finally, we connect our findings to the phenomenon of drag reduction in turbulent flows. Since the total dissipation can be computed just from the knowledge of the velocity profile at the walls, any amount of drag reduction must be reflected by a corresponding reduction of the gradient at the walls. Concurrently, the energy intake by the fluctuations from the mean flow should reduce as well. Indeed, the latter effect was measured in both experiments [9] and simulations [10,11]. The question is which is the chicken and which is the egg. In our calculation we identified that the reduction in production comes first. From Figs. 4 and 5 (upper panel) which are at the same value of Re we see that the dissipation does not change at all when the mixing layer moves, but the production is strongly affected. Of course, at steady state the velocity gradient at the wall must adjust as shown in Fig. 5 (lower panel). We recognize that in a turbulent flow there are a number of modes that interact, but we propose that a similar mechanism operates for each mode at its critical layer, where both elastic and viscous effect determine the mean flow. In the present calculation we can consider all the putative unstable modes, and conclude that with a viscosity gradient similar to that seen in polymeric turbulent flows the threshold Re goes up five times (to 31000). We leave the confirmation of this prediction to future experiments.

Acknowledgments. This work has been supported by the Israel Science Foundation.

[1] R.H. Nadolink and W.W. Haigh, ASME Appl. Mech. Rev. 48, 351 (1995).

[2] J. L. Lumley, Ann. Rev. Fluid Mech. 1, 367 (1969).

[3] K.R. Sreenivasan and C. M. White, J. Fluid Mech., 409, 149 (2000).

[4] M. Tabor and P.G. de Gennes, Europhys. Lett., 2, 519 (1986).

[5] P.G. de Gennes, Introduction to Polymer Dynamics, (Cambridge, 1990).

[6] B. T. Ranganathan and R. Govindarajan, Phys. Fluids 13, 1 (2001).

[7] R. Sureshkumar, A. N. Beris and R.A. Handler, Phys. Fluids 9, 743 (1997).

[8] F.M White, Viscous Fluid Flow (McGraw Hill, 1991).

[9] J.M.J den Toonder, M.A. Hulsen,G.D.C Kuiken and F.T.M Niewstadt, J. Fluid Mech. 337, 193 (1997).

[10] C.D. Dimitropoulos, R. Sureshkumar, A.N. Beris and R.A. Handler, Phys. Fluids, 13, 1016 (2001).

[11] E. De Angelis, "The Influence of Polymer Additives on the Structure of Wall Turbulence", Thesis, Universita' di Roma, La Sapienza, 2000. 